\title{
Descending thoracic aorta to femoral bypass grafting for total abdominal aortic occlusion
}

\author{
Olgar Bayserke1, Yavuz Beşoğul ${ }^{2}$ \\ ${ }^{1}$ Department of Cardiovascular Surgery, Medical Faculty of Maltepe University, İstanbul, Turkey \\ ${ }^{2}$ Department of Cardiovascular Surgery, Medicana Hospital, İstanbul, Turkey
}

Received: October 20, 2015 Accepted: December 19, 2015 Published online: January 11, 2017

\section{ABSTRACT}

Descending thoracic aorta to femoral bypass graft is an alternative procedure for revascularization of the lower extremities to conventional techniques, when the abdominal aorta is complete obstructed. In general, this technique is performed by surgeons, when abdomen is unable to be opened due to the presence of an abdominal infection, fistulas, or hostile abdomen. In this article, we present our four-year outcomes of descending thoracic aorta to femoral bypass with cross-femoral bypass in four patients. There was no mortality and all grafts were patent. Descending thoracic aorta to femoral bypass grafting has excellent overall long-term results.

Keywords: Abdominal aortic occlusion; aortofemoral bypass; descending thoracic.

The thoracic aorta to femoral bypass grafting is an ideal procedure for a small subgroup of patients, comprising those with an occluded old aorta to femoral bypass graft, those with a lead-pipe calcified infrarenal aorta which is unsuitable as an inflow source, and those with a hostile abdomen (i.e., ileal conduit, ileostomy or colostomy or a previous aortic graft infection). Complete obstruction of the abdominal aorta at the renal artery level is often a surgical challenge in case of a long-standing thrombosis, short suprarenal aortic space, and extensive periarterial inflammatory reaction. Therefore, a descending thoracic aorta to femoral bypass graft is used as an alternative for revascularization. Herein, we present our four-year outcomes of descending thoracic aorta to femoral bypass grafting with cross-femoral bypass in four patients.

\section{SURGICAL TECHNIQUE}

Descending thoracic aorta to femoral bypass grafting was performed in four patients who had a juxtarenal complete obstruction of the abdominal aorta in our hospital (Figure 1). All patients were males with a mean age of 65.5 (range, 59-72) years and they had severe atherosclerotic disease and hypercholesterolemia. None of them was, however, diabetic. They had claudication and ischemic pain of the lower extremity. Two of them were ex-smokers with normal pulmonary function test results [Forced expiratory volume in one second $\left.\left(\mathrm{FEV}_{1}\right) \geq 75\right]$. The patients had no renal disease, and biochemical screening results were normal. Coronary angiography revealed coronary atherosclerosis in only one patient. Coronary artery bypass surgery grafting was performed by off-pump technique to this patient before three months.

A written informed consent was obtained from each patient. Selective intubation with double-lumen tubes were performed under fentanyl anesthesia. The patients were positioned to position the hip flat; however, the torso was slightly rotated to the right. Four incisions were made: a left posterolateral thoracotomy, two groin incisions, and a lateral abdominal incision. The descending thoracic aorta was approached through the thoracotomy at the level of the sixth-seventh intercostal space. The left lung was decompressed by selective intubation, and the aorta was cleanly dissected. Common femoral arteries were obtained via two groin incisions. The psoas were reached through the lateral abdominal incisions and with an extra-peritoneal approach. A retroperitoneal tunnel was, then, fashioned for the passage of the

Corresponding author: Olgar Bayserke, MD. Maltepe Üniversitesi Tip Fakültesi Hastanesi Kalp ve Damar Cerrahisi Anabilim Dall, 34844 Maltepe, İstanbul, Turkey.

Tel: +90 505 - 2525778 e-mail: olgarbayserke@yahoo.com 
graft. Once an $8 \mathrm{~mm}$ silver, INTERGARD collagencoated knitted polyester vascular prosthesis graft was carried from this tunnel, the patient underwent systemic heparinization $(100 \mathrm{U} / \mathrm{kg})$. The proximal end of the graft was reached the through the aortic hiatus at the diaphragm level. When the blood pressure was reduced by vasodilators, the descending thoracic aorta was controlled with side-clamp, and an end-to-side anastomosis was performed. The distal end of the graft was fashioned with an endto-side anastomosis to the left common femoral artery. An $8 \mathrm{~mm}$ INTERGARD collagen-coated knitted polyester vascular prosthesis graft was, then, performed through the left femoral to the right femoral crossover bypass. The side-aortic clamp was removed and the grafts were visualized with pulsing. Finally, after the bleeding was controlled, the lung was re-inflated, and the chest was closed in a standard fashion over a chest tube.

There was no postoperative mortality. Except one patient, all were extubated in the operation room without a need for respiratory support. However, one patient required respiratory support for six hours. There were no pleural effusion; however, two patients had pulmonary atelectasis for four days. Oral antiaggregant (acetylsalicylic acid) treatment was started in the postoperative third day, following prophylaxis of lowmolecular-weight heparin in the postoperative early

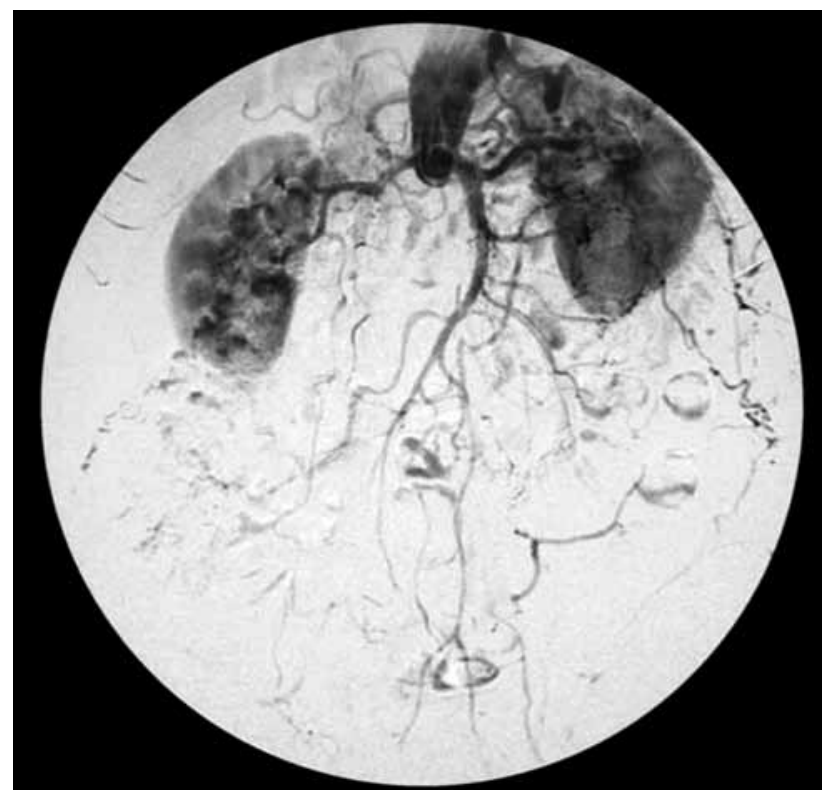

Figure 1. Preoperatively angiography of 60-year-old male patient. period. The mean length of intensive care and hospital stays was two days and eight days, respectively. Distal pulses of the lower extremities were sensed at discharge. The graft failure was not seen within the first month of follow-up. Computed tomography (CT) angiography revealed that all grafts were patent at four years (Figure 2).

\section{DISCUSSION}

Bypass grafting which originate from the descending thoracic aorta to femoral arteries are well-described, although they are not commonly used in the first-line setting. The descending thoracic aorta to femoral bypass grafting was first described in 1961 in two cases. ${ }^{[1]}$ The thoracic aorta is a good inflow option, as its exposure is relatively straight forward and this segment is usually free of significant atherosclerosis. ${ }^{[2]}$ Most authors have suggested that the use of the descending thoracic aorta with a retroperitoneal tunnel yields more acceptable operative risks and patency rates. Five year patency rates of the procedure has been reported as $86 \%$ in the literature. ${ }^{[3]}$

Axillobifemoral bypass is the most common first choice as an alternative to aortofemoral incomplete

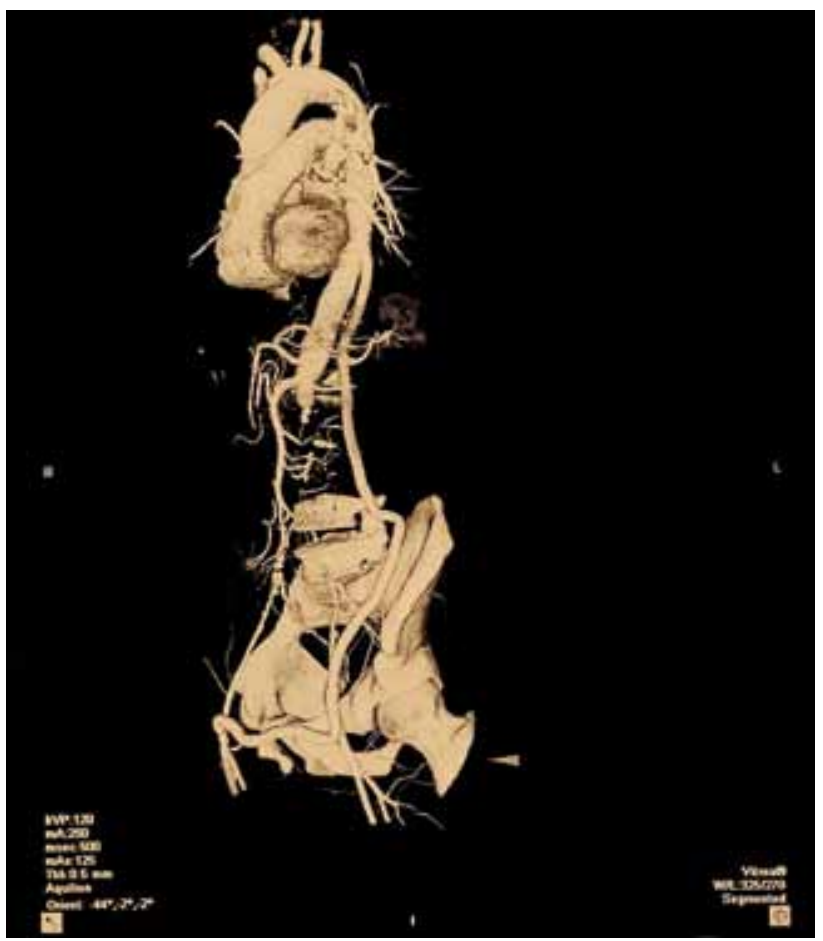

Figure 2. Control computed tomographic angiography of 60-year-old male patient in postoperative first mount. 
obstruction of the abdominal aorta at the level of renal arteries. ${ }^{[4]}$ However, the long-term patency is lower, due to the longer graft length with potential compression along the superficial course. ${ }^{[1]}$

In our technique, we used the descending thoracic aorta, rather than the axillary artery. In most reports, the bifurcated graft is favored to thoracofemoral and femorofemoral crossover grafts. ${ }^{[5,6]}$ We used this graft in only one patient.

Furthermore, thoracic aorta is controlled either with a side-biting clamp or with two completely occluding aortic clamps placed in close proximity to each other. One of the disadvantages of completely occluding clamping is that renal circulation can be impaired, if the time of proximal anastomosis to aorta is hold over, and spinal cord and mesenteric ischemia can develop. Partial aortic control with sideclamping is successful in most cases, although it is not recommended, when the aorta is heavily diseased and calcified. Additionally, it makes the anastomosis more difficult, as the margins of aortotomy become closely opposed. ${ }^{[1]}$ A partial side-clamp was placed in our patients, and it did not complicate the anastomosis.

Baird et al. ${ }^{[6]}$ described ascending aorta to femoral bypass grafting through a median sternotomy. The advantage of this technique is that concomitant coronary revascularization can be performed, when indicated. One of our patients who had coronary artery disease underwent offpump coronary artery bypass grafting three months before thoracofemoral grafting. Subsequently, we performed revascularization of the lower extremities by descending thoracic aorta to femoral and femorofemoral crossover bypass grafting.

In the present study, all patients were eligible descending thoracic aorta to femoral bypass grafting with excellent overall long-term results. Despite pulmonary difficulties, we believe that this procedure is a satisfactory alternative for the treatment of juxtarenal complete abdominal aortic occlusions, as the thoracic aorta is no more atherosclerotic, and there is high blood flow in this segment to the axillary artery.

In conclusion, endovascular treatment of infrarenal aortic occlusions is feasible in most cases with favorable midterm patency rates. However, due to relatively high procedurally-related complication rates, cautious selection of the patients and taking appropriate preventive measures are necessary to improve the immediate outcomes. ${ }^{[7]}$

\section{Declaration of conflicting interests}

The authors declared no conflicts of interest with respect to the authorship and/or publication of this article.

\section{Funding}

The authors received no financial support for the research and/or authorship of this article.

\section{REFERENCES}

1. Stevenson JK, Sauvage LR, Harkins HR. A bypass homograft from thoracic aorta to femoral arteries for occlusive vascular disease: case report. Ann Surg 1961;27:632-7.

2. Kalman PG. Thoracofemoral bypass:A Useful Addition to a vascular Surgeon's Armamentarium.Perspectives in vascular Surgery and Endovascular Therapy 2004;16:59-64.

3. McCarthy WJ, Mesh CL, McMillan WD, Flinn WR, Pearce WH, Yao JS. Descending thoracic aorta-to-femoral artery bypass: ten years' experience with a durable procedure. J Vasc Surg 1993;17:336-47.

4. Köksal C, Sarikaya S, Zengin M. Thoracofemoral bypass for treatment of juxtarenal aortic occlusion. Asian Cardiovasc Thorac Ann 2002;10:141-4.

5. Haas KL, Moulder PV, Kerstein MD. Use of thoracic aortobifemoral artery bypass grafting as an alternative procedure for occlusive aortoiliac disease. Am Surg 1985;51:573-6.

6. Baird RJ, Ropchan GV, Oates TK, Weisel RD, Provan JL. Ascending aorta to bifemoral bypass--a ventral aorta. J Vasc Surg 1986;3:405-10.

7. Kim TH, Ko YG, Kim U, Kim JS, Choi D, Hong MK, et al. Outcomes of endovascular treatment of chronic total occlusion of the infrarenal aorta. J Vasc Surg 2011;53:1542-9. 